\title{
TRIPARTITE GAME MODELS IN A DUAL-CHANNEL SUPPLY CHAIN: COMPETITION AND COOPERATION
}

\author{
Zhenkai Lou ${ }^{1}$, Fujun Hou ${ }^{1, *}$, Xuming Lou ${ }^{2}$ and Yubing Zhai ${ }^{1}$
}

\begin{abstract}
This paper considers tripartite games in a dual-channel supply chain which involves a manufacturer, an offline retailer and an online retailer. Both competition and cooperation issues are analyzed. In the competition model, a Stackelberg game between the manufacturer and two retailers and a Bertrand game between two retailers occur simultaneously. It is shown that the channel which attracts more consumers' purchase preference is charged a higher wholesale price and it meanwhile declares a higher sales price. In the presence of revenue sharing, cooperation issues between the three participants are studied and the change of the revenue of each participant is analyzed when partial cooperation exists. Further, the definition of the optimum two-player coalition is proposed. We demonstrate that the channel which attracts more preference of consumers is definitely in the optimum coalition. The structure of the two-player coalition is analyzed. Finally, under revenue sharing and cost apportionments, the change of each participant's profit is examined.
\end{abstract}

Mathematics Subject Classification. 90B50, 93A30.

Received June 25, 2020. Accepted February 20, 2021.

\section{INTRODUCTION}

With the development of Internet technique, more and more people prefer to purchase through the e-commerce platform. Owing to this, many manufacturers redesign their traditional channel structures by engaging in direct sales [5]. The selling channel brought by the Internet endows the manufacturers with more opportunities for cost savings, revenue growth, and expansion to new market segments [4]. Because of various preference of consumers, demands on the traditional retail channel and the online channel are always different under a same retail price. It is proved that the channel preference plays a critical role in pricing decisions of manufacturers and retailers [14].

In dual-channel supply chains, pricing issues always involve multiple participants. In order to possess high bargaining power, some participant may look for an appropriate cooperator. When a contract between the same type of participants (manufacturers or retailers) reaches, a crosswise alliance forms with the purpose of enhancing the revenue of each participant in it [20]. In other cases, vertical alliances may form to acquire more bargaining power. There are many examples in practice with regard to this point. For instance, Wal-Mart

Keywords. Tripartite games, competition and cooperation, optimum two-player coalition, equal distribution criterion.

1 School of Management and Economics, Beijing Institute of Technology, Beijing, P.R. China.

2 School of Economics and Management, Xi'an University of Posts and Telecommunications, Xi'an, P.R. China.

${ }^{*}$ Corresponding author: houf j@bit.edu.cn 
chooses Procter \& Gamble as its cooperator to form a vertical alliance. In other situations, the online channel may cooperate with physical stores so as to reduce its delivery cost $[7,26]$. For the members in an alliance, they can share information and jointly make decisions to gain a higher total revenue. However, few literatures discussed the superiority of a crosswise alliance or a vertical alliance till now.

Inspired by the above phenomenon, we explore competition and cooperation issues in a dual-channel supply chain. In practice, a manufacturer sometimes is involved in the competition between different channel retailers. For instance, there are a supermarket and an online platform (e.g., Dmall) in a certain region, both of which sell fresh products to consumers in this region. When a manufacturer sells dairy products or vegetables to the two channel retailers, a Stackelberg game occurs between the three participants.

This paper reveals some theoretic results and highlights some managerial insights. Firstly, it is shown that the channel retailer who possesses more consumers' preference gains more expected profits. Secondly, it is demonstrated that the revenue of one participant definitely decreases when the other two choose to cooperate. Following that, the concept of an optimal two-player alliance is proposed to deal with the case in which not all of the participants can jointly make decisions on account of some reasons. Finally, an allocation based on the equal distribution criterion is proposed. In the tripartite cooperation case, we demonstrate that the members in the optimal two-player alliance are dominant when allocating the total profits. In practice, issues of how an alliance forms and expands are significant, as well as the change of each participant's profit.

The reminder of this paper is organized as follows. Section 2 presents some literature related to this paper, and shows the main contributions. Section 3 introduces the notation and constructs the objective functions for all the participants. In Section 4, a competition tripartite game is conducted. In Section 5, we consider the cooperation issues between the three participants, and allocate the profit for each participant according to the priority of cooperation. Section 6 provides a numerical illustration to verify the obtained conclusions under different price-sensitive coefficients. Section 7 summarizes the paper. All proofs of this paper are in the appendices.

\section{LiterATURE REVIEW}

There is a growing body of literature on hunting optimal prices for dual-channel supply chains. Paper [22] considered channel conflict issues when involving an e-commerce channel, and demonstrated a number of counterintuitive structural properties. Paper [24] did a significant work on determining competitive pricing policies between the brick-and-mortar retail channel and the online distribution channel. Paper [6] proposed a model in which consumers chose the purchase channel based on both price and service qualities. Paper [9] developed a pricing-and-production integrated model for a dual-channel supply chain. Paper [21] considered both competition and coordination issues between dual sales channels in a supply chain. Sometimes, a manufacturer manages dual sales channels, and he has to trade off all costs and revenues in this situation [18].

The above literature mostly considers dual-channel issues as two-person decision problems, which sometimes may not coincide with practical cases. For example, the online channel, just like the traditional retail channel, may price its product independently. In this case, both offline and online channels participate in making decisions, just similar to the discussion in [10]. Paper [23] discussed a tripartite Stackelberg game in which the retailer is the leader and two manufacturers are followers. Paper [16] studied competition and cooperation issues in a dual-channel supply chain consisting of one manufacturer and two retailers, which is similar to our framework. In addition, paper [11] studied game models of dual channels involving one manufacturer and multiple retailers.

In spite of a large number of researches related to pricing issues on dual-channel supply chains, there is a lack of discussion on the superiority of a crosswise alliance and a vertical alliance. For the purpose of maximizing the profit, the participants will look for their optimal cooperator. This paper shows that the members in the optimum two-player coalition possess the superiority when allocating the final profits, which is a research gap. In addition, we propose a judging criterion to determine which two-player alliance is the optimal, which is of significance for a dual-channel supply chain when dealing with the selection of the optimal cooperator. 
TABLE 1. Notation definition.

\begin{tabular}{ll}
\hline \hline Symbol & Description \\
\hline$a$ & Potential product demands of the market for the product \\
$\lambda$ & Proportion of the offline channel's market share \\
$1-\lambda$ & Proportion of the online channel's market share \\
$\delta$ & Expected marginal demand with respect to the sales price \\
$\theta$ & Shift between the two channels with regards to the price \\
$p_{m}$ & Wholesale price by which the manufacturer sells to the offline retailer \\
$p_{n}$ & Wholesale price by which the manufacturer sells to the online retailer \\
$p_{r}$ & Retail price of the offline channel determined by the offline retailer \\
$p_{s}$ & Retail price of the online channel determined by the online retailer \\
$d_{r}$ & Expected demand in the offline channel, $d_{r}=\lambda a-\delta p_{r}+\theta p_{s}$ \\
$d_{s}$ & Expected demand in the online channel, $d_{s}=(1-\lambda) a-\delta p_{s}+\theta p_{r}$ \\
$Z$ & Total expected revenue of the manufacturer \\
$U$ & Expected revenue of the offline retailer \\
$V$ & Expected revenue of the online retailer \\
\hline
\end{tabular}

The framework of this paper is similar to paper [8]. We construct both a decentralized model and a centralized model for the dual-channel supply chain. A Stackelberg game between the manufacturer and two retailers and a Bertrand game between two retailers occur simultaneously in the competition case. In the cooperation case, joint pricing always occurs by coordinating between different participants $[1,12]$. Besides, the dominance of the manufacturer or the retailer was examined in the pricing issues of the dual-channel supply chain [15]. Different from the existing literature, we examine the optimal two-player coalition in the cooperative situation, by considering the fact that any two participants' cooperation incurs a reduction on the revenue of the other one. The superiority of the vertical alliance and the crosswise alliance is compared. In addition, paper [25] examined the change on the price incurred by cooperation. Our research yields more results at this point of view.

To be specific, this paper discusses the following problems. In the competition case, the manufacturer determines optimal wholesale prices for the two channels, aiming to maximize its total revenue. Then the offline retailer and the online retailer declare their retail prices simultaneously. Optimal prices and revenues of the two retailers are compared. While considering cooperative issues, the total profit is assumed to be transferable, i.e., we investigate transferable utility games, just similar to $[17,21]$. Because the revenue of one participant changes when the other two participants choose to cooperate, classical allocation methods may not be applicable. In this paper, we propose an allocation method based on the equal distribution criteria by considering the number of participants to allocate the increased profit. The allocation approach guarantees the vested interest for those who have formed an alliance.

\section{Notations And ASSUMPtions}

In this paper, the selling period is finite and the expected demand refers to the expected sales volume within this period. A single product is sold and shortage is not permitted. All the participants acquire complete information. Quality problems are not concerned and sales return is not considered either.

Consider a two-echelon supply chain which involves a manufacturer, an online retailer, and an offline retailer. The notations used throughout the paper are given as Table 1:

The coefficient of each channel's market share follows [3,13]. Besides, by [2] we know that $\delta \geq \theta$ always holds so as to guarantee $d_{r}+d_{s} \leq a$. Because the potential product demand $a$ cannot be reached for $p_{r}>0$ and $p_{s}>0$, we further assume that $\delta>\theta$ to enhance the rate of the decline in total demands with respect to the sales prices. 
Given the above notations and assumptions, the objective function of the manufacturer with $p_{m}$ and $p_{n}$ as its decision variables is

$$
Z=p_{m} d_{r}+p_{n} d_{s}
$$

Similarly, the objective function of the offline retailer is formulated as follows:

$$
U=\left(p_{r}-p_{m}\right) d_{r}
$$

And the objective function of the online retailer with $p_{s}$ as its decision variable is as follows:

$$
V=\left(p_{s}-p_{n}\right) d_{s} .
$$

For the sake of following discussion, some premises are given as follows:

(1) The production capacity of the manufacturer is assumed to be sufficient to meet the market demand, i.e., this paper doesn't consider stock-out issues.

(2) In practice, the inventory cost sometimes is a fixed fee - mainly refers to facilities costs and house rental costs, which doesn't affect the participants' decision. Following this scenario, this paper doesn't take the inventory cost into account.

\section{Competition tripartite-Game model}

This section concerns competition issues among a manufacturer, an online retailer and an offline retailer within a known finite selling period. The manufacturer is required to determine wholesale prices first, and then the offline retailer and the online retailer declare their retail prices simultaneously. We inversely examine the models to obtain the equilibrium solution.

According to the decision order, the manufacturer needs to take into account the decisions of the two retailers, while either of the retailers only needs to consider the other retailer's decision. By these analyses, the involved model is presented.

The model of the manufacturer for acquiring optimal wholesale prices $p_{m}$ and $p_{n}$ is given as follows:

$$
\begin{aligned}
& \max _{p_{m}, p_{n}} Z=p_{m}\left(\lambda a-\delta p_{r}+\theta p_{s}\right)+p_{n}\left[(1-\lambda) a-\delta p_{s}+\theta p_{r}\right] \\
& \text { s.t. } p_{r} \in \arg \max U, p_{s} \in \arg \max V .
\end{aligned}
$$

The model of the offline retailer with $p_{r}$ as its decision variable is

$$
\max _{p_{r}} U=\left(p_{r}-p_{m}\right)\left(\lambda a-\delta p_{r}+\theta p_{s}\right) .
$$

Meanwhile, the model of the online retailer with $p_{s}$ as its decision variable is

$$
\left.\max _{p_{s}} V=\left(p_{s}-p_{n}\right)\left[(1-\lambda) a-\delta p_{s}+\theta p_{r}\right)\right] .
$$

Clearly, the manufacturer is the leader and two retailers are followers. Hence, a Stackelberg game consisting of the above three models is presented. In addition, model (4.2) and model (4.3) compose a Bertrand game, without considering the decision variables of the manufacturer.

By differentiating $U$, we obtain the following equation:

$$
\frac{\partial U}{\partial p_{r}}=-2 \delta p_{r}+\theta p_{s}+\delta p_{m}+\lambda a=0 .
$$

Examining the second-order derivative of $U$, we have

$$
\frac{\partial^{2} U}{\partial p_{r}^{2}}=-2 \delta<0
$$


which means that the solution of equation (4.4) is the unique solution of $\max U$.

Similarly, the corresponding equation of $V$ is

$$
\frac{\partial V}{\partial p_{s}}=-2 \delta p_{s}+\theta p_{r}+\delta p_{n}+(1-\lambda) a=0,
$$

the solution of which is the unique solution of $\max V$.

We denote by $p_{r}^{*}$ and $p_{s}^{*}$ the solutions of model (4.2) and model (4.3), respectively. The solutions of $p_{r}^{*}$ and $p_{s}^{*}$ are acquired by handling the above two equations:

$$
\left\{\begin{array}{l}
p_{r}^{*}=\frac{2 \delta^{2} p_{m}+\delta \theta p_{n}+2 \delta \lambda a+\theta(1-\lambda) a}{4 \delta^{2}-\theta^{2}} \\
p_{s}^{*}=\frac{\delta \theta p_{m}+2 \delta^{2} p_{n}+2 \delta(1-\lambda) a+\theta \lambda a}{4 \delta^{2}-\theta^{2}}
\end{array} .\right.
$$

Apparently, (4.5) is the unique equilibrium solution of the discussed Bertrand game.

The objective function of $Z$ is then obtained by substituting solutions (4.5) into the objective function of model (4.1):

$$
\begin{aligned}
Z= & -\frac{2 \delta^{3}-\delta \theta^{2}}{4 \delta^{2}-\theta^{2}} p_{m}^{2}-\frac{2 \delta^{3}-\delta \theta^{2}}{4 \delta^{2}-\theta^{2}} p_{n}^{2}+\frac{2 \delta^{2} \theta}{4 \delta^{2}-\theta^{2}} p_{m} p_{n} \\
& +\frac{2 \delta^{2} \lambda a+\delta \theta(1-\lambda) a}{4 \delta^{2}-\theta^{2}} p_{m}+\frac{2 \delta^{2}(1-\lambda) a+\delta \theta \lambda a}{4 \delta^{2}-\theta^{2}} p_{n}
\end{aligned}
$$

Differentiating $Z$, we obtain the partial derivatives of $Z$. Letting them both be equal to zero, we have

$$
\left\{\begin{array}{l}
\frac{\partial Z}{\partial p_{m}}=-\frac{2\left(2 \delta^{3}-\delta \theta^{2}\right)}{4 \delta^{2}-\theta^{2}} p_{m}+\frac{2 \delta^{2} \theta}{4 \delta^{2}-\theta^{2}} p_{n}+\frac{2 \delta^{2} \lambda a+\delta \theta(1-\lambda) a}{4 \delta^{2}-\theta^{2}}=0 \\
\frac{\partial Z}{\partial p_{n}}=-\frac{2\left(2 \delta^{3}-\delta \theta^{2}\right)}{4 \delta^{2}-\theta^{2}} p_{n}+\frac{2 \delta^{2} \theta}{4 \delta^{2}-\theta^{2}} p_{m}+\frac{2 \delta^{2}(1-\lambda) a+\delta \theta \lambda a}{4 \delta^{2}-\theta^{2}}=0
\end{array}\right.
$$

Denoting by $p_{m}^{*}$ and $p_{n}^{*}$ the solution of equation set (4.7), we have

$$
\left\{\begin{array}{l}
p_{m}^{*}=\frac{8 \delta^{3} \lambda a+8 \delta^{2} \theta(1-\lambda) a-2 \delta \theta^{2} \lambda a-2 \theta^{3}(1-\lambda) a}{\left(4 \delta^{2}-2 \theta^{2}\right)^{2}-4 \delta^{2} \theta^{2}} \\
p_{n}^{*}=\frac{8 \delta^{3}(1-\lambda) a+8 \delta^{2} \theta \lambda a-2 \delta \theta^{2}(1-\lambda) a-2 \theta^{3} \lambda a}{\left(4 \delta^{2}-2 \theta^{2}\right)^{2}-4 \delta^{2} \theta^{2}}
\end{array} .\right.
$$

In fact, by recombining factors in the denominator, we have

$$
\left(4 \delta^{2}-2 \theta^{2}\right)^{2}-4 \delta^{2} \theta^{2}=16 \delta^{4}-20 \delta^{2} \theta^{2}+4 \theta^{4}=4\left(\delta^{2}-\theta^{2}\right)\left(4 \delta^{2}-\theta^{2}\right),
$$

by which (4.8) is transformed to

$$
\left\{\begin{array}{l}
p_{m}^{*}=\frac{\delta \lambda a+\theta(1-\lambda) a}{2 \delta^{2}-2 \theta^{2}} \\
p_{n}^{*}=\frac{\delta(1-\lambda) a+\theta \lambda a}{2 \delta^{2}-2 \theta^{2}}
\end{array} .\right.
$$

The Hessian matrix of $Z$ given by function (4.6)

$$
H=\left[\begin{array}{ll}
-\frac{2\left(2 \delta^{3}-\delta \theta^{2}\right)}{4 \delta^{2}-\theta^{2}} & \frac{2 \delta^{2} \theta}{4 \delta^{2}-\theta^{2}} \\
\frac{2 \delta^{2} \theta}{4 \delta^{2}-\theta^{2}} & -\frac{2\left(2 \delta^{3}-\delta \theta^{2}\right)}{4 \delta^{2}-\theta^{2}}
\end{array}\right]
$$

is clearly negative definite by considering $\delta>\theta>0$, which implies that solution (4.8) is the unique solution of $\max Z$ given by model (4.1). Hence, the Stackelberg game also has a unique equilibrium solution. 
By substituting (4.8) into (4.5), the values of $p_{r}^{*}$ and $p_{s}^{*}$ are then acquired:

$$
\left\{\begin{array}{l}
p_{r}^{*}=\frac{6 \delta^{3} \lambda a+5 \delta^{2} \theta(1-\lambda) a-3 \delta \theta^{2} \lambda a-2 \theta^{3}(1-\lambda) a}{\left(4 \delta^{2}-\theta^{2}\right)\left(2 \delta^{2}-2 \theta^{2}\right)} \\
p_{s}^{*}=\frac{6 \delta^{3}(1-\lambda) a+5 \delta^{2} \theta \lambda a-3 \delta \theta^{2}(1-\lambda) a-2 \theta^{3} \lambda a}{\left(4 \delta^{2}-\theta^{2}\right)\left(2 \delta^{2}-2 \theta^{2}\right)}
\end{array}\right.
$$

If the offline channel attracts more preference of consumers, i.e., $\lambda>1 / 2$, it is obtained that

$$
p_{m}^{*}-p_{n}^{*}=\frac{(\delta-\theta)(2 \lambda-1) a}{2 \delta^{2}-2 \theta^{2}}>0
$$

and

$$
\begin{aligned}
p_{r}^{*}-p_{s}^{*} & =\frac{\left(6 \delta^{3}-5 \delta^{2} \theta-3 \delta \theta^{2}+2 \theta^{3}\right)(2 \lambda-1) a}{\left(4 \delta^{2}-\theta^{2}\right)\left(2 \delta^{2}-2 \theta^{2}\right)} \\
& >\frac{\left(6 \delta^{3}-6 \delta^{2} \theta-3 \delta \theta^{2}+3 \theta^{3}\right)(2 \lambda-1) a}{\left(4 \delta^{2}-\theta^{2}\right)\left(2 \delta^{2}-2 \theta^{2}\right)} \\
& =\frac{\left(6 \delta^{2}-3 \theta^{2}\right)(\delta-\theta)(2 \lambda-1) a}{\left(4 \delta^{2}-\theta^{2}\right)\left(2 \delta^{2}-2 \theta^{2}\right)} \\
& >0,
\end{aligned}
$$

which suggest that the wholesale price of the offline retailer determined by the manufacturer and the retail price of the offline retailer are both higher than the online retailer's ones.

Similarly, if the online channel attracts more consumers' preference, i.e., by $0<\lambda<1 / 2$, we have

$$
p_{n}^{*}-p_{m}^{*}=\frac{(\delta-\theta)(1-2 \lambda) a}{2 \delta^{2}-2 \theta^{2}}>0
$$

and

$$
\begin{aligned}
p_{s}^{*}-p_{r}^{*} & =\frac{\left(6 \delta^{3}-5 \delta^{2} \theta-3 \delta \theta^{2}+2 \theta^{3}\right)(1-2 \lambda) a}{\left(4 \delta^{2}-\theta^{2}\right)\left(2 \delta^{2}-2 \theta^{2}\right)} \\
& >\frac{\left(6 \delta^{3}-6 \delta^{2} \theta-3 \delta \theta^{2}+3 \theta^{3}\right)(1-2 \lambda) a}{\left(4 \delta^{2}-\theta^{2}\right)\left(2 \delta^{2}-2 \theta^{2}\right)} \\
& >0,
\end{aligned}
$$

which imply that the wholesale price of the online retailer and the retail price of the online retailer are higher in this situation.

Given the above, we have the following conclusion:

Proposition 4.1. For the channel which possesses more consumers' preference, both the wholesale price given by the manufacturer and the sales price declared by the channel itself are higher than the other channel.

The above conclusion reveals a fact that, the channel retailer which possesses more consumers' preference often declares a higher sales price to gain more profits. While for the other channel retailer, a lower sales price is more reasonable for guaranteeing a certain sale quantity.

By solution (4.8) and solution (4.9), the value of $\max Z$ is obtained:

$$
\max Z=\frac{2 \delta^{3} \lambda^{2} a^{2}+2 \delta^{3}(1-\lambda)^{2} a^{2}+6 \delta^{2} \theta \lambda(1-\lambda) a^{2}+\delta \theta^{2} \lambda^{2} a^{2}+\delta \theta^{2}(1-\lambda)^{2} a^{2}}{\left(4 \delta^{2}-\theta^{2}\right)\left(4 \delta^{2}-4 \theta^{2}\right)} .
$$


Similarly, the value of $\max U$ is

$$
\max U=\frac{\delta[2 \delta \lambda+\theta(1-\lambda)]^{2} a^{2}}{4\left(4 \delta^{2}-\theta^{2}\right)^{2}}
$$

and the value of $\max V$ is as follows:

$$
\max V=\frac{\delta[2 \delta(1-\lambda)+\theta \lambda]^{2} a^{2}}{4\left(4 \delta^{2}-\theta^{2}\right)^{2}} .
$$

Comparing the value of $\max U$ and the value of $\max V$, if $\lambda>1 / 2$, we have

$$
\max U-\max V=\frac{[(2 \delta-\theta)(2 \lambda-1)](2 \delta+\theta) \delta a^{2}}{4\left(4 \delta^{2}-\theta^{2}\right)^{2}}>0 .
$$

Otherwise, $\max U-\max V<0$. Hence, we have the following conclusion:

Proposition 4.2. The channel retailer who possesses more consumers' preference definitely gains more expected revenue.

In practice, a high sales quantity doesn't always lead to a high profit. The above conclusion makes sense only in the discussed framework.

\section{CoOperation tripartite-game models}

In this section, the cooperative behavior among the three participants is considered under the premise that revenues are transferable. The changes of optimal prices are analyzed. Moreover, an allocation method based on the equal distribution criteria by considering the number of participants to allocate the increased profit.

In practice, the two sales channels sometimes are not able to cooperate because they may both try to grab the market share. In this situation, the manufacture is entitled to choose a better cooperator. Without loss of generality, we first consider the cooperation between the manufacturer and the offline retailer. In this situation, the wholesale price $p_{m}$ no longer exists [19]. The corresponding model for maximizing their aggregate expected revenue is

$$
\begin{aligned}
& \max (Z+U)=p_{r}\left(\lambda a-\delta p_{r}+\theta p_{s}\right)+p_{n}\left[(1-\lambda) a-\delta p_{s}+\theta p_{r}\right] \\
& \quad p_{n}, p_{r} \\
& \text { s.t. } p_{s} \in \arg \max V .
\end{aligned}
$$

The model of the online retailer is still (4.3). In order to make a distinction, we denote by $V^{\prime}$ the expected revenue of the online retailer in this section, and $p_{s}^{*}$ the solutions of model of model (5.1). Then the expression of solution of model (4.3) is

$$
p_{s}^{*}=\frac{(1-\lambda) a+\theta p_{r}+\delta p_{n}}{2 \delta},
$$

by which the objective function of model (5.1) is transformed to

$$
Z+U=-\frac{2 \delta^{2}-\theta^{2}}{2 \delta} p_{r}^{2}-\frac{\delta}{2} p_{n}^{2}+\theta p_{r} p_{n}+\frac{2 \delta \lambda a+\theta(1-\lambda) a}{2 \delta} p_{r}+\frac{(1-\lambda) a}{2} p_{n} .
$$

Differentiating $(Z+U)$ and letting the partial derivatives both be equal to zero, we have

$$
\left\{\begin{array}{l}
\frac{\partial(Z+U)}{\partial p_{r}}=-\frac{2 \delta^{2}-\theta^{2}}{\delta} p_{r}+\theta p_{n}+\frac{2 \delta \lambda a+\theta(1-\lambda) a}{2 \delta}=0 \\
\frac{\partial(Z+U)}{\partial p_{n}}=-\delta p_{n}+\theta p_{r}+\frac{(1-\lambda) a}{2}=0
\end{array} .\right.
$$


Denoting by $p_{r}^{*}$ and $p_{n}^{*}$ the solution of equation set (5.3), it is then obtained

$$
\left\{\begin{array}{l}
p_{r}^{*}=\frac{\delta \lambda a+\theta(1-\lambda) a}{2 \delta^{2}-2 \theta^{2}} \\
p_{n}^{*}=\frac{\delta(1-\lambda) a+\theta \lambda a}{2 \delta^{2}-2 \theta^{2}}
\end{array} .\right.
$$

Examining the Hessian matrix of equation set (5.3),

$$
H=\left[\begin{array}{ll}
-\frac{2 \delta^{2}-\theta^{2}}{\delta} & \theta \\
\theta & -\delta
\end{array}\right]
$$

is negative definite by considering $\delta>\theta$, which implies that solution (5.4) is the unique solution to $\max (Z+U)$.

By solution (5.4), we have

$$
p_{s}^{*}=\frac{3 \delta^{2}(1-\lambda) a+2 \delta \theta \lambda a-\theta^{2}(1-\lambda) a}{2 \delta\left(2 \delta^{2}-2 \theta^{2}\right)} .
$$

Substituting solution (5.4) and solution (5.5) into function (5.2), the solution of model (5.1) is then acquired:

$$
\max (Z+U)=\frac{2 \delta^{2} \lambda^{2} a^{2}+\delta^{2}(1-\lambda)^{2} a^{2}+4 \delta \theta \lambda(1-\lambda) a^{2}+\theta^{2}(1-\lambda)^{2} a^{2}}{4 \delta\left(2 \delta^{2}-2 \theta^{2}\right)} .
$$

And the value of $\max V^{\prime}$ is

$$
\max V^{\prime}=\frac{(1-\lambda)^{2} a^{2}}{16 \delta}
$$

Next, values of $(\max Z+\max U)$ and $\max (Z+U)$ are compared. The first one is determined in the tripartite competition game, and the second one is determined in the competition-and-cooperation mixed game.

In order to be convenient, we first compare $\max (Z+U)$ and $\max Z$ and simplify as follows:

$$
\max (Z+U)-\max Z=\frac{[2 \delta \lambda+\theta(1-\lambda)]^{2} a^{2}}{8 \delta\left(4 \delta^{2}-\theta^{2}\right)} .
$$

Then we have

$$
\begin{aligned}
\max (Z+U)-\max Z-\max U & =\frac{\left(4 \delta^{2}-\theta^{2}\right)\left[2 \delta \lambda+\theta(1-\lambda)^{2}\right] a^{2}}{8 \delta\left(4 \delta^{2}-\theta^{2}\right)^{2}}-\frac{2 \delta^{2}[2 \delta \lambda+\theta(1-\lambda)]^{2} a^{2}}{8 \delta\left(4 \delta^{2}-\theta^{2}\right)^{2}} \\
& =\frac{\left(4 \delta^{2}-2 \delta^{2}-\theta^{2}\right)[2 \delta \lambda+\theta(1-\lambda)]^{2} a^{2}}{8 \delta\left(4 \delta^{2}-\theta^{2}\right)^{2}} \\
& >0 .
\end{aligned}
$$

By the above result, it is shown that the total revenue of the manufacturer and the offline retailer is definitely higher when they jointly make decisions in the tripartite game, i.e.,

$$
\max (Z+U)>\max Z+\max U .
$$

We call the value of $(\max (Z+U)-\max Z-\max U)$ the additional revenue increased by cooperation.

Comparing solution (4.12) and solution (5.7), we have

$$
\begin{aligned}
\max V^{\prime}-\max V & =\frac{(1-\lambda)^{2} a^{2}}{16 \delta}-\frac{\delta\left[2 \delta(1-\lambda)+\theta \lambda^{2}\right] a^{2}}{4\left(4 \delta^{2}-\theta^{2}\right)^{2}} \\
& <\frac{(1-\lambda)^{2} a^{2}}{16 \delta}-\frac{\delta[2 \delta(1-\lambda)]^{2} a^{2}}{4 \times 16 \delta^{4}} \\
& =0,
\end{aligned}
$$


which suggests that the online retailer gains less expected revenue when the manufacturer and the offline retailer cooperate to make their decisions.

According to the equal distribution criterion of the additional revenue increased by cooperation, the expected revenue of the manufacturer $Z_{1}$ is

$$
Z_{1}=\max Z+\frac{\max (Z+U)-\max Z-\max U}{2}=\frac{\max (Z+U)+\max Z-\max U}{2} .
$$

Similarly, the expected revenue of the offline retailer $U_{1}$ is

$$
U_{1}=\frac{\max (Z+U)-\max Z+\max U}{2} .
$$

Besides, $V_{1}=\max V^{\prime}$.

Based on the symmetry property between the revenue functions of the two retailers, the cooperation between the manufacturer and the online retailer is analyzed by the same way, and some similar results are obtained. We use the same notations to denote optimal prices and present the results as follows.

Based on the coalition of the manufacturer and the online retailer, a bi-level framework with objective functions $\max (Z+V)$ and $\max U$ is formulated. The optimal prices of the coalition are

$$
\left\{\begin{array}{l}
p_{s}^{*}=\frac{\delta(1-\lambda) a+\theta \lambda a}{2 \delta^{2}-2 \theta^{2}} \\
p_{m}^{*}=\frac{\delta \lambda a+\theta(1-\lambda) a}{2 \delta^{2}-2 \theta^{2}}
\end{array} .\right.
$$

The optimal price of the offline retailer is

$$
p_{r}^{*}=\frac{3 \delta^{2} \lambda a+2 \delta \theta(1-\lambda) a-\theta^{2} \lambda a}{2 \delta\left(2 \delta^{2}-2 \theta^{2}\right)} .
$$

The expected revenue of the coalition is

$$
\max (Z+V)=\frac{2 \delta^{2}(1-\lambda)^{2} a^{2}+\delta^{2} \lambda^{2} a^{2}+4 \delta \theta \lambda(1-\lambda) a^{2}+\theta^{2} \lambda^{2} a^{2}}{4 \delta\left(2 \delta^{2}-2 \theta^{2}\right)} .
$$

And the value of $\max U^{\prime}$ is

$$
\max U^{\prime}=\frac{\lambda^{2} a^{2}}{16 \delta}
$$

According to the equal distribution criterion of the additional revenue increased by cooperation, the expected revenue of the manufacturer $Z_{2}$ is

$$
Z_{2}=\frac{\max (Z+V)+\max Z-\max V}{2}
$$

Similarly, the expected revenue of the online retailer $V_{2}$ is

$$
V_{2}=\frac{\max (Z+V)-\max Z+\max V}{2} .
$$

Besides, $U_{2}=\max U^{\prime}$. It is easy to verify that $\max U^{\prime}<\max U$ by comparing the values of (4.11) and (5.13). Similar to the previous situation, we can obtain the following results by the same process:

$$
\max (Z+V)>\max Z+\max V
$$

and

$$
\max U^{\prime}<\max U
$$


Next, the coalition of the two retailers is considered when they have willingness to cooperate.

The model of the manufacturer is still model (4.1). In order to make a distinction, we denote by $Z^{\prime}$ the expected revenue of the manufacturer here. In order to figure out it, the model of the two-retailers coalition needs to be analyzed first.

The model for maximizing their aggregate expected revenue is

$$
\underset{p_{r}, p_{s}}{\max }(U+V)=\left(p_{r}-p_{m}\right)\left(\lambda a-\delta p_{r}+\theta p_{s}\right)+\left(p_{s}-p_{n}\right)\left[(1-\lambda) a-\delta p_{s}+\theta p_{r}\right] .
$$

Differentiating $(U+V)$ and letting the partial derivatives both be equal to zero, we have

$$
\left\{\begin{array}{l}
\frac{\partial(U+V)}{\partial p_{r}}=-2 \delta p_{r}+2 \theta p_{s}+\lambda a+\delta p_{m}-\theta p_{n}=0 \\
\frac{\partial(U+V)}{\partial p_{s}}=-2 \delta p_{s}+2 \theta p_{r}+(1-\lambda) a+\delta p_{n}-\theta p_{m}=0
\end{array} .\right.
$$

Denoting by $p_{r}^{*}$ and $p_{s}^{*}$ the solution of equation set (5.18). Solving it, we have

$$
\left\{\begin{array}{l}
p_{r}^{*}=\frac{p_{m}}{2}+\frac{\delta \lambda a+\theta(1-\lambda) a}{2 \delta^{2}-2 \theta^{2}} \\
p_{s}^{*}=\frac{p_{n}}{2}+\frac{\delta(1-\lambda) a+\theta \lambda a}{2 \delta^{2}-2 \theta^{2}}
\end{array},\right.
$$

which is the unique solution of equation set (5.17) on the basis of the negative definite of the Hessian matrix of $(U+V)$ :

$$
H=\left[\begin{array}{ll}
-2 \delta & 2 \theta \\
2 \theta & -2 \delta
\end{array}\right] .
$$

By expressions (5.18), the objective function of model (4.1) is transformed to

$$
Z^{\prime}=-\frac{\delta}{2} p_{m}^{2}-\frac{\delta}{2} p_{n}^{2}+\theta p_{m} p_{n}+\frac{\lambda a}{2} p_{m}+\frac{(1-\lambda) a}{2} p_{n}
$$

Letting the partial derivatives of $Z^{\prime}$ both be equal to zero, we have

$$
\left\{\begin{array}{l}
\frac{\partial Z^{\prime}}{\partial p_{m}}=-\delta p_{m}+\theta p_{n}+\frac{\lambda a}{2}=0 \\
\frac{\partial Z^{\prime}}{\partial p_{n}}=-\delta p_{n}+\theta p_{m}+\frac{(1-\lambda) a}{2}=0
\end{array} .\right.
$$

Denoting by $p_{m}^{*}$ and $p_{n}^{*}$ the solution of equation set (5.20), we have

$$
\left\{\begin{array}{l}
p_{m}^{*}=\frac{\delta \lambda a+\theta(1-\lambda) a}{2 \delta^{2}-2 \theta^{2}} \\
p_{n}^{*}=\frac{\delta(1-\lambda) a+\theta \lambda a}{2 \delta^{2}-2 \theta^{2}}
\end{array} .\right.
$$

Then $p_{r}^{*}$ and $p_{s}^{*}$ are obtained by substituting solution (5.21) into expressions (5.18):

$$
\left\{\begin{array}{l}
p_{r}^{*}=\frac{3 \delta \lambda a+3 \theta(1-\lambda) a}{4 \delta^{2}-4 \theta^{2}} \\
p_{s}^{*}=\frac{3 \delta(1-\lambda) a+3 \theta \lambda a}{4 \delta^{2}-4 \theta^{2}}
\end{array} .\right.
$$

The value of $\max (U+V)$ is acquired as follows by solution (5.21) and solution (5.22):

$$
\max (U+V)=\frac{\delta \lambda^{2} a^{2}+\delta(1-\lambda)^{2} a^{2}+2 \theta \lambda(1-\lambda) a^{2}}{16\left(\delta^{2}-\theta^{2}\right)} .
$$

Moreover, the expected revenue of the manufacturer is as follows:

$$
\max Z^{\prime}=\frac{\delta \lambda^{2} a^{2}+\delta(1-\lambda)^{2} a^{2}+2 \theta \lambda(1-\lambda) a^{2}}{8\left(\delta^{2}-\theta^{2}\right)} .
$$


Comparing $\max (U+V)$ and $(\max U+\max V)$, we have

$$
\begin{aligned}
\max (U+V)-\max U-\max V & =\frac{\left(4 \delta^{3} \theta^{2}+5 \delta \theta^{4}\right) \lambda^{2} a^{2}+\left(16 \delta^{2} \theta^{3}+2 \theta^{5}\right) \lambda(1-\lambda) a^{2}+\left(4 \delta^{3} \theta^{2}+5 \delta \theta^{4}\right)(1-\lambda)^{2} a^{2}}{16\left(\delta^{2}-\theta^{2}\right)\left(4 \delta^{2}-\theta^{2}\right)^{2}} \\
& >0 .
\end{aligned}
$$

Comparing $\max Z$ and $\max Z^{\prime}$, we have

$$
\begin{aligned}
\max Z & =\frac{2 \delta^{3} \lambda^{2} a^{2}+2 \delta^{3}(1-\lambda)^{2} a^{2}+6 \delta^{2} \theta \lambda(1-\lambda) a^{2}+\delta \theta^{2} \lambda^{2} a^{2}+\delta \theta^{2}(1-\lambda)^{2} a^{2}}{\left(4 \delta^{2}-\theta^{2}\right)\left(4 \delta^{2}-4 \theta^{2}\right)} \\
& >\frac{2 \delta^{3} \lambda^{2} a^{2}+2 \delta^{3}(1-\lambda)^{2} a^{2}+6 \delta^{2} \theta \lambda(1-\lambda) a^{2}}{16 \delta^{2}\left(\delta^{2}-\theta^{2}\right)} \\
& >\frac{\delta \lambda^{2} a^{2}+\delta(1-\lambda)^{2} a^{2}+2 \theta \lambda(1-\lambda) a^{2}}{8\left(\delta^{2}-\theta^{2}\right)}=\max Z^{\prime} .
\end{aligned}
$$

According to the equal distribution criterion, the expected revenue of the offline retailer $U_{3}$ is

$$
U_{3}=\frac{\max (U+V)+\max U-\max V}{2} .
$$

The expected revenue of the online retailer $V_{3}$ is

$$
V_{3}=\frac{\max (U+V)-\max U+\max V}{2} .
$$

In addition, $Z_{3}=\max Z^{\prime}$.

Given all the above results, we draw some meaningful conclusions as follows:

Proposition 5.1. Under the equal distribution criterion of the additional revenue increased by cooperation, any two participants enhance their individual profits by cooperation.

Proposition 5.2. Any two participants' cooperation incurs a reduction on the revenue of the other one.

Actually, the bargaining power of the noncooperative participant suffers a loss when the other two participants form an alliance.

Next, the optimum two-player coalition is examined based on the equal distribution criterion of the additional revenue increased by cooperation.

If the offline channel attracts more consumers' preference, i.e., $\lambda>1 / 2$, it is obtained that $\max U>\max V$ according to Proposition 4.2. Comparing the additional revenues increased by cooperation, we obtain

$$
\begin{aligned}
{[\max (Z+U)-\max Z-\max U]-} & {[\max (Z+V)-\max Z-\max V] } \\
= & {[\max (Z+U)-\max (Z+V)]-(\max U-\max V) } \\
= & \frac{\left[\delta^{2} \lambda^{2}-\theta^{2} \lambda^{2}-\delta^{2}(1-\lambda)^{2}+\theta^{2}(1-\lambda)^{2}\right] a^{2}}{4 \delta\left(2 \delta^{2}-2 \theta^{2}\right)} \\
& -\frac{\left[4 \delta^{3} \lambda^{2}-\delta \theta^{2} \lambda^{2}-4 \delta^{3}(1-\lambda)^{2}+\delta \theta^{2}(1-\lambda)^{2}\right] a^{2}}{4\left(4 \delta^{2}-\theta^{2}\right)^{2}} \\
= & \frac{\lambda^{2} a^{2}-(1-\lambda)^{2} a^{2}}{8 \delta}-\frac{\delta \lambda^{2} a^{2}-\delta(1-\lambda)^{2} a^{2}}{4\left(4 \delta^{2}-\theta^{2}\right)} \\
> & \frac{\delta \lambda^{2} a^{2}-\delta(1-\lambda)^{2} a^{2}}{8 \delta^{2}}-\frac{\delta \lambda^{2} a^{2}-\delta(1-\lambda)^{2} a^{2}}{12 \delta^{2}} \\
> & 0,
\end{aligned}
$$


which implies that the manufacturer gains more additional revenues by cooperating with the offline retailer.

If $\lambda<1 / 2, \max V>\max U$ holds according to Proposition 4.2. Then we have

$$
\begin{aligned}
{[\max (Z+V)-\max Z-\max V] } & -[\max (Z+U)-\max Z-\max U] \\
& =[\max (Z+V)-\max (Z+U)]-(\max V-\max U) \\
& =\frac{(1-\lambda)^{2} a^{2}-\lambda^{2} a^{2}}{8 \delta}-\frac{\delta(1-\lambda)^{2} a^{2}-\delta \lambda^{2} a^{2}}{4\left(4 \delta^{2}-\theta^{2}\right)} \\
& >\frac{\delta(1-\lambda)^{2} a^{2}-\delta \lambda^{2} a^{2}}{8 \delta^{2}}-\frac{\delta(1-\lambda)^{2} a^{2}-\delta \lambda^{2} a^{2}}{12 \delta^{2}} \\
& >0,
\end{aligned}
$$

which means that the manufacturer gains more additional revenues by cooperating with the online retailer.

Given the above, we have the following conclusion:

Proposition 5.3. The manufacturer always chooses the channel retailer who attracts more consumers' preference to form a coalition.

Of course, the retailer who attracts more consumers' preference naturally considers that whether the eventually revenue would be higher by cooperating with the other retailer instead of the manufacturer.

Without loss of generality, the offline retailer is assumed to be the one who attracts more preference of consumers in the following discussion (the handling is similar when the online channel attracts more preference of consumers). Then it is required to consider the following formula:

$$
[\max (U+V)-\max U-\max V]-[\max (Z+U)-\max Z-\max U] .
$$

If it is positive, the offline retailer prefers to choose the online retailer to form a two-player coalition. Otherwise, the manufacturer is its optimal option. Moreover, the value of formula (5.27) depends on $\lambda, \delta$ and $\theta$. We show the proof in the Appendix A.

For the two-player coalition, we propose the following definition:

Definition 5.4. A two-player coalition is deemed to be optimal, if and only if both of the two participants gain more revenues in this coalition than in other coalitions.

In view of the above discussion, the following conclusion is drawn:

Proposition 5.5. The retail channel which attracts more consumers' preference is one member of the optimum two-player coalition.

Although the more attractive retail channel has the priority to choose a cooperator, the optimal cooperator is certain for any given case. Given this point, we hold the opinion that the members in the optimal two-player coalition are of equal importance. Hence, the allocation method based on the equal distribution criteria is reasonable for allocating the increased profit.

The one, the manufacturer or the retail channel with less attraction, who is not in the two-player coalition, pursues to cooperate with the coalition so as to enhance his revenue. However, he has less bargaining power than the optimal two-player coalition. In order to reflect this point, we use the allocation method based on the equal distribution criteria by considering the number of participants to allocate the increased profit.

The model for maximizing their aggregate expected revenue is

$$
\max \left(\underset{p_{r}, p_{s}}{Z}+U\right)=p_{r}\left(\lambda a-\delta p_{r}+\theta p_{s}\right)+p_{s}\left[(1-\lambda) a-\delta p_{s}+\theta p_{r}\right] .
$$


Differentiating $(Z+U+V)$ and letting the partial derivatives be equal to zero, we have

$$
\left\{\begin{array}{l}
\frac{\partial(Z+U+V)}{\partial p_{r}}=-2 \delta p_{r}+2 \theta p_{s}+\lambda a=0 \\
\frac{\partial(Z+U+V)}{\partial p_{s}}=-2 \delta p_{s}+2 \theta p_{r}+(1-\lambda) a=0
\end{array} .\right.
$$

The solution of equation set (5.29) is

$$
\left\{\begin{array}{l}
p_{r}^{*}=\frac{\delta \lambda a+\theta(1-\lambda) a}{2 \delta^{2}-2 \theta^{2}} \\
p_{s}^{*}=\frac{\delta(1-\lambda) a+\theta \lambda a}{2 \delta^{2}-2 \theta^{2}}
\end{array},\right.
$$

which is the unique solution by considering the negative definite of the Hessian matrix of $(U+V)$ :

$$
H=\left[\begin{array}{ll}
-2 \delta & 2 \theta \\
2 \theta & -2 \delta
\end{array}\right]
$$

By solution (5.30), the value of $\max (Z+U+V)$ is obtained:

$$
\max (Z+U+V)=\frac{\delta \lambda^{2} a^{2}+\delta(1-\lambda)^{2} a^{2}+2 \theta \lambda(1-\lambda) a^{2}}{4 \delta^{2}-4 \theta^{2}} .
$$

Clearly, both

$$
\max (Z+U+V)>\max (Z+U)+\max V^{\prime}
$$

and

$$
\max (Z+U+V)>\max (U+V)+\max Z
$$

hold as the solution given by formula (5.30) is the unique solution of $\max (Z+U+V)$.

Hence, we have the following results by the equal distribution criterion:

(1) If $(Z, U)$ is the optimum two-player coalition, the ultima revenue of the manufacturer is

$$
\begin{aligned}
Z^{*} & =\frac{\max (Z+U)+\max Z-\max U}{2}+\frac{\max (Z+U+V)-\max (Z+U)-\max V^{\prime}}{3} \\
& =\frac{2 \max (Z+U+V)+\max (Z+U)+3 \max Z-3 \max U-2 \max V^{\prime}}{6} .
\end{aligned}
$$

Similarly, the eventual revenue of the offline retailer is

$$
U^{*}=\frac{2 \max (Z+U+V)+\max (Z+U)-3 \max Z+3 \max U-2 \max V^{\prime}}{6} .
$$

And the revenue of the online retailer is

$$
V^{*}=\frac{\max (Z+U+V)-\max (Z+U)+2 \max V^{\prime}}{3} .
$$

(2) If $(U, V)$ is the optimum two-player coalition, the final revenue of the manufacturer is

$$
Z^{*}=\frac{\max (Z+U+V)-\max (U+V)+2 \max Z^{\prime}}{3} .
$$

The ultima revenue of the offline retailer is

$$
\begin{aligned}
U^{*} & =\frac{\max (U+V)+\max U-\max V}{2}+\frac{\max (Z+U+V)-\max Z^{\prime}-\max (U+V)}{3} \\
& =\frac{2 \max (Z+U+V)+\max (U+V)-2 \max Z^{\prime}+3 \max U-3 \max V}{6} .
\end{aligned}
$$

Similarly, the eventual revenue of the online retailer is

$$
V^{*}=\frac{2 \max (Z+U+V)+\max (U+V)-2 \max Z^{\prime}-3 \max U+3 \max V}{6} .
$$




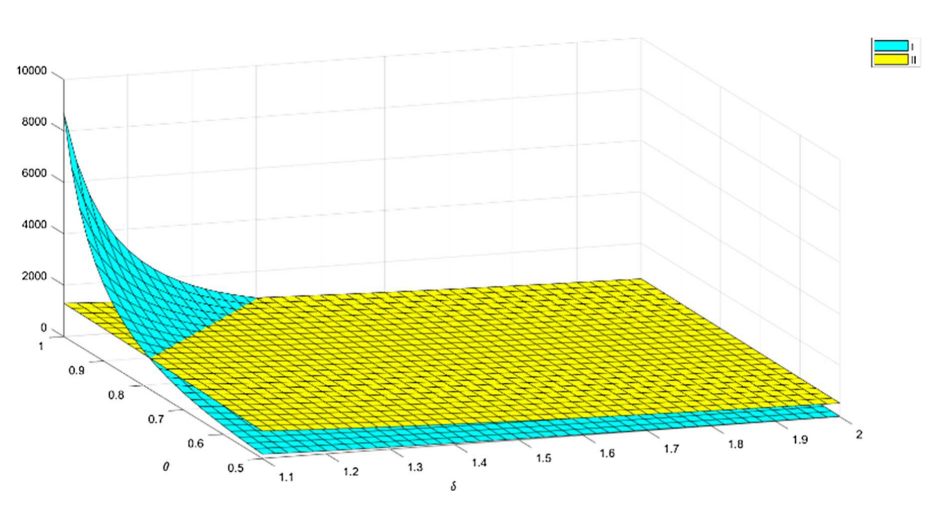

FIGURE 1. Surfaces of the two increased profit functions.

Obviously, the members of the optimum two-player alliance always gain more revenues than they earn in the competition situation. But for the other participant who is not in the alliance, we have following conclusions for the above two situations (the proof is shown in the Appendix B):

(1) When $(Z, U)$ is the optimum two-player coalition, the difference between $V^{*}$ and $\max V$ depends on $\delta, \theta$, and $\lambda$.

(2) When $(U, V)$ is the optimum two-player coalition, the difference between $Z^{*}$ and $\max Z$ also depends on $\delta$, $\theta$, and $\lambda$.

\section{A NUMERICAL EXAMPLE}

This section provides a numerical illustration to verify the obtained conclusions under different price-sensitive coefficients. Consider the following scenario: $a=200, \lambda=0.6, \delta \in[1.1,2], \theta \in[0.5,1]$.

Clearly, according to proposition 6 , the offline retailer is one member of the optimal two-player coalition. The function surfaces are as follows, where I represents $[\max (U+V)-\max U-\max V]$, and II represents $[\max (Z+U)-\max Z-\max U]:$ (Fig. 1)

It is shown by the graph that the two channel retailers may form a coalition first only when the two pricesensitive coefficients are close.

Next, we take a sample to see how the pricing alliance forms, and compare the profit of the last member in the tripartite competition model and in the centralized decision model.

Given $a=200, \lambda=0.6, \delta=2$ and $\theta=1$. Apparently, the offline retailer is one member of the optimal two-player coalition. Then we focus on finding out the other member. Because

$$
[\max (U+V)-\max U-\max V]-[\max (Z+U)-\max Z-\max U]=139.6-609.8=-470.2,
$$

the manufacturer is the other member of the optimal two-player coalition (see Fig. 2 below).

We show the following line chart to describe the change of online retailer's profit, where point 1 represents the profit in the tripartite competition case, point 2 represents the profit in the case when the optimal two-player coalition forms, and point 3 represents the profit in the case when the online retailer joins in the coalition.

By the above illustration, we know that the profit of online retailer in the big alliance is lower than the one in the tripartite competition situation. However, if he doesn't join the alliance, his profit must be lower. 


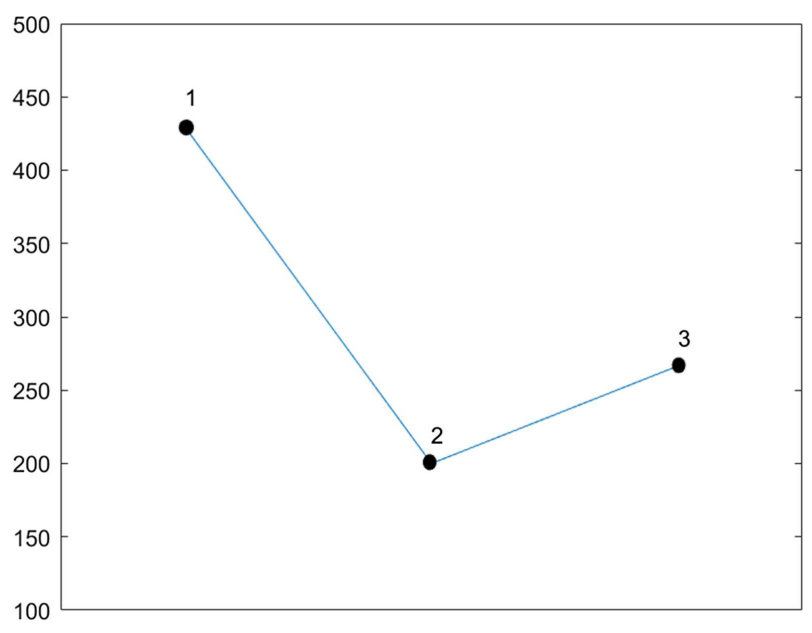

FiguRE 2. The change of online retailer's profit in different situations.

\section{Conclusions}

In this paper, we investigate tripartite games in a dual-channel supply chain under both competition and cooperation situations.

In the competition situation, a Stackelberg game between the manufacturer and two retailers and a Bertrand game between two retailers are involved. The uniqueness of all the optimal prices is demonstrated. The importance of the channel preference is highlighted. It is shown that the channel which attracts more consumers' purchasing preference is charged a higher wholesale price by the manufacturer and the channel meanwhile declares a higher sales price.

In the cooperation situation, the fact that any two participants' cooperation incurs a reduction on the revenue of the other one is revealed. The equal distribution criterion of the additional revenue increased by cooperation is proposed to allocate the aggregate revenue. Following that, the issue of an optimum two-player coalition is discussed.

This paper hasn't considered the difference of both the expected marginal demands and the shift coefficients for the two channels, which is a limitation of the current research. Actually, consumers may have different sensibilities for the sales prices on different sales channels. Some different results may be obtained by considering this point. Besides, in order to avoid a great deal of computation, stock-holding costs, ordering costs, and distribution costs are not considered. In our following research, we will take more factors into consideration when dealing with dual-channel pricing issues.

\section{Appendix A.}

The proof for the following conclusion: the value of

$$
[\max (U+V)-\max U-\max V]-[\max (Z+U)-\max Z-\max U]
$$

depends on $\delta$ and $\theta$, where $\lambda>1 / 2$.

Proof. By the obtained results, we have

$$
\max (U+V)=\frac{\delta \lambda^{2} a^{2}+\delta(1-\lambda)^{2} a^{2}+2 \theta \lambda(1-\lambda) a^{2}}{16\left(\delta^{2}-\theta^{2}\right)}
$$


668

$$
\begin{aligned}
= & \frac{16 \delta^{5} \lambda^{2} a^{2}+16 \delta^{5}(1-\lambda)^{2} a^{2}+32^{4} \delta \theta \lambda(1-\lambda) a^{2}}{16\left(\delta^{2}-\theta^{2}\right)\left(4 \delta^{2}-\theta^{2}\right)^{2}}+\frac{\delta \theta^{4} \lambda^{2} a^{2}+\delta \theta^{4}(1-\lambda)^{2} a^{2}+2 \theta^{5} \lambda(1-\lambda) a^{2}}{16\left(\delta^{2}-\theta^{2}\right)\left(4 \delta^{2}-\theta^{2}\right)^{2}} \\
& -\frac{8 \delta^{3} \theta^{2} \lambda^{2} a^{2}+8 \delta^{3} \theta^{2}(1-\lambda)^{2} a^{2}+16 \delta^{2} \theta^{3} \lambda(1-\lambda) a^{2}}{16\left(\delta^{2}-\theta^{2}\right)\left(4 \delta^{2}-\theta^{2}\right)^{2}}, \\
\max U= & \frac{\delta[2 \delta \lambda+\theta(1-\lambda)]^{2} a^{2}}{4\left(4 \delta^{2}-\theta^{2}\right)^{2}}=\frac{16 \delta^{5} \lambda^{2} a^{2}+16 \delta^{4} \theta \lambda(1-\lambda) a^{2}+4 \delta^{3} \theta^{2}(1-\lambda) a^{2}}{16\left(\delta^{2}-\theta^{2}\right)\left(4 \delta^{2}-\theta^{2}\right)^{2}} \\
& -\frac{16 \delta^{3} \theta^{2} \lambda^{2} a^{2}+16 \delta^{2} \theta^{3} \lambda(1-\lambda) a^{2}+4 \delta \theta^{4}(1-\lambda)^{2} a^{2}}{16\left(\delta^{2}-\theta^{2}\right)\left(4 \delta^{2}-\theta^{2}\right)^{2}},
\end{aligned}
$$

and

$$
\begin{aligned}
\max V= & \frac{\delta[2 \delta(1-\lambda)+\theta \lambda]^{2} a^{2}}{4\left(4 \delta^{2}-\theta^{2}\right)^{2}}=\frac{16 \delta^{5}(1-\lambda)^{2} a^{2}+16 \delta^{4} \theta \lambda(1-\lambda) a^{2}+4 \delta^{3} \theta^{2} \lambda^{2} a^{2}}{16\left(\delta^{2}-\theta^{2}\right)\left(4 \delta^{2}-\theta^{2}\right)^{2}} \\
& -\frac{16 \delta^{3} \theta^{2}(1-\lambda)^{2} a^{2}+16 \delta^{2} \theta^{3} \lambda(1-\lambda) a^{2}+4 \delta \theta^{4} \lambda^{2} a^{2}}{16\left(\delta^{2}-\theta^{2}\right)\left(4 \delta^{2}-\theta^{2}\right)^{2}} .
\end{aligned}
$$

Afterwards, it is obtained that

$$
\begin{aligned}
\max (U+V) & -\max U-\max V \\
& =\frac{4 \delta^{3} \theta^{2}\left[\lambda^{2}+(1-\lambda)^{2}\right] a^{2}+16 \delta^{2} \theta^{3} \lambda(1-\lambda) a^{2}+5 \delta \theta^{4}\left[\lambda^{2}+(1-\lambda)^{2}\right] a^{2}+2 \theta^{5} \lambda(1-\lambda) a^{2}}{16\left(\delta^{2}-\theta^{2}\right)\left(4 \delta^{2}-\theta^{2}\right)^{2}} .
\end{aligned}
$$

In addition, for the other additional value, we have

$$
\begin{aligned}
\max (Z+U)= & \frac{2 \delta^{2} \lambda^{2} a^{2}+\delta^{2}(1-\lambda)^{2} a^{2}+4 \delta \theta \lambda(1-\lambda)^{2} a^{2}+\theta^{2}(1-\lambda)^{2} a^{2}}{4 \delta\left(2 \delta^{2}-2 \theta^{2}\right)} \\
= & \frac{32 \delta^{6} \lambda^{2} a^{2}+16 \delta^{6}(1-\lambda)^{2} a^{2}+64 \delta^{5} \theta \lambda(1-\lambda) a^{2}-16 \delta^{4} \theta^{2} \lambda^{2} a^{2}+8 \delta^{4} \theta^{2}(1-\lambda)^{2} a^{2}}{8 \delta\left(\delta^{2}-\theta^{2}\right)\left(4 \delta^{2}-\theta^{2}\right)^{2}} \\
& -\frac{32 \delta^{3} \theta^{3} \lambda(1-\lambda) a^{2}-2 \delta^{2} \theta^{4} \lambda^{2} a^{2}+7 \delta^{2} \theta^{4}(1-\lambda)^{2} a^{2}-4 \delta^{5} \theta \lambda(1-\lambda) a^{2}-\theta^{6}(1-\lambda)^{2} a^{2}}{8 \delta\left(\delta^{2}-\theta^{2}\right)\left(4 \delta^{2}-\theta^{2}\right)^{2}}, \\
\max Z= & \frac{2 \delta^{3} \lambda^{2} a^{2}+2 \delta^{3}(1-\lambda)^{2} a^{2}+6 \delta^{2} \theta \lambda(1-\lambda) a^{2}+\delta \theta^{2} \lambda^{2} a^{2}+\delta \theta^{2}(1-\lambda)^{2} a^{2}}{\left(4 \delta^{2}-\theta^{2}\right)(4 \delta-4 \theta)} \\
= & \frac{16 \delta^{6} \lambda^{2} a^{2}+16 \delta^{6}(1-\lambda)^{2} a^{2}+48 \delta^{5} \theta \lambda(1-\lambda) a^{2}+4 \delta^{4} \theta^{2} \lambda^{2} a^{2}+4 \delta^{4} \theta^{2}(1-\lambda)^{2} a^{2}}{8 \delta\left(\delta^{2}-\theta^{2}\right)\left(4 \delta^{2}-\theta^{2}\right)^{2}} \\
& -\frac{12 \delta^{3} \theta^{3} \lambda(1-\lambda) a^{2}+2 \delta^{2} \theta^{4} \lambda^{2} a^{2}+2 \delta^{2} \theta^{4}(1-\lambda)^{2} a^{2}}{8 \delta\left(\delta^{2}-\theta^{2}\right)\left(4 \delta^{2}-\theta^{2}\right)^{2}},
\end{aligned}
$$

and

$$
\begin{aligned}
\max U= & \frac{\delta[2 \delta \lambda+\theta(1-\lambda)]^{2} a^{2}}{4\left(4 \delta^{2}-\theta^{2}\right)^{2}} \\
= & \frac{8 \delta^{6} \lambda^{2} a^{2}+8 \delta^{5} \theta \lambda(1-\lambda) a^{2}+2 \delta^{4} \theta^{2}(1-\lambda)^{2} a^{2}}{8 \delta\left(\delta^{2}-\theta^{2}\right)\left(4 \delta^{2}-\theta^{2}\right)^{2}} \\
& -\frac{8 \delta^{4} \theta^{2} \lambda^{2} a^{2}+8 \delta^{3} \theta^{3} \lambda(1-\lambda) a^{2}+2 \delta^{2} \theta^{4}(1-\lambda)^{2} a^{2}}{8 \delta\left(\delta^{2}-\theta^{2}\right)\left(4 \delta^{2}-\theta^{2}\right)^{2}} .
\end{aligned}
$$


Then we obtain that

$$
\begin{aligned}
\max (Z+U)- & \max Z-\max U \\
= & \frac{8 \delta^{6} \lambda^{2} a^{2}+8 \delta^{5} \theta \lambda(1-\lambda) a^{2}+2 \delta^{4} \theta^{2}(1-\lambda)^{2} a^{2}-12 \delta^{4} \theta^{2} \lambda^{2} a^{2}-12 \delta^{3} \theta^{3} \lambda(1-\lambda) a^{2}}{8 \delta\left(\delta^{2}-\theta^{2}\right)\left(4 \delta^{2}-\theta^{2}\right)^{2}} \\
& +\frac{4 \delta^{2} \theta^{4} \lambda^{2} a^{2}-3 \delta^{2} \theta^{4}(1-\lambda)^{2} a^{2}+4 \delta \theta^{5} \lambda(1-\lambda) a^{2}+\theta^{3}(1-\lambda)^{2} a^{2}}{8 \delta\left(\delta^{2}-\theta^{2}\right)\left(4 \delta^{2}-\theta^{2}\right)^{2}} \\
= & \frac{\left[8 \delta^{4} \lambda^{2}-4 \delta^{2} \theta^{2} \lambda^{2}+8 \delta^{3} \theta \lambda(1-\lambda)-4 \delta \theta^{3} \lambda(1-\lambda)+2 \delta^{2} \theta^{2}(1-\lambda)^{2}-\theta^{4}(1-\lambda)^{2}\right] a^{2}}{8 \delta\left(4 \delta^{2}-\theta^{2}\right)} .
\end{aligned}
$$

It is easy to find that the value of $[\max (U+V)-\max U-\max V]$ will be very large when the difference between $\delta$ and $\theta$ is small enough. By contrast, the value of $[\max (Z+U)-\max Z-\max U]$ isn't affected directly by the difference between $\delta$ and $\theta$. Hence, when the difference between $\delta$ and $\theta$ is smaller than a certain threshold value, the value of $[\max (U+V)-\max U-\max V]$ will be larger than the value of $[\max (Z+U)-\max Z-\max U]$.

Moreover, by comparing the highest order items in (A.1) and (A.2), we find that the value of $[\max (Z+U)-$ $\max Z-\max U]$ grows faster than the value of $[\max (U+V)-\max U-\max V]$ with the increase on the difference between $\delta$ and $\theta$.

For example, when $\delta=2 \theta$, (A.1) is transformed to

$$
\begin{aligned}
\max (Z+U) & -\max Z-\max U \\
& =\frac{\left[8 \delta^{4} \lambda^{2}-4 \delta^{2} \theta^{2} \lambda^{2}+8 \delta^{3} \theta \lambda(1-\lambda)-4 \delta^{3} \theta \lambda(1-\lambda)+2 \delta^{2} \theta^{2}(1-\lambda)^{2}-\theta^{4}(1-\lambda)^{2}\right] a^{2}}{8 \delta\left(4 \delta^{2}-\theta^{2}\right)^{2}} \\
& =\frac{3 \theta^{2}\left[16 \delta^{4} \lambda^{2}-8 \delta^{2} \theta^{2} \lambda^{2}+16 \delta^{3} \theta \lambda(1-\lambda)-8 \delta \theta^{3} \lambda(1-\lambda)+4 \delta^{2} \theta^{2}(1-\lambda)^{2}-2 \theta^{4}(1-\lambda)^{2}\right] a^{2}}{16 \delta\left(\delta^{2}-\theta^{2}\right)\left(4 \delta^{2}-\theta^{2}\right)^{2}} .
\end{aligned}
$$

By $\delta>\theta$, we have

$$
\begin{aligned}
\max (Z+U) & -\max Z-\max U \\
& =\frac{3 \theta^{2}\left[16 \delta^{4} \lambda^{2}-8 \delta^{2} \theta^{2} \lambda^{2}+16 \delta^{3} \theta \lambda(1-\lambda)-8 \delta \theta^{3} \lambda(1-\lambda)+4 \delta^{2} \theta^{2}(1-\lambda)^{2}-2 \theta^{4}(1-\lambda)^{2}\right] a^{2}}{16 \delta\left(\delta^{2}-\theta^{2}\right)\left(4 \delta^{2}-\theta^{2}\right)^{2}} \\
& >\frac{3 \theta^{2}\left[8 \delta^{4} \lambda^{2}+8 \delta^{3} \theta \lambda(1-\lambda)+2 \delta^{2} \theta^{2}(1-\lambda)^{2}\right] a^{2}}{16 \delta\left(\delta^{2}-\theta^{2}\right)\left(4 \delta^{2}-\theta^{2}\right)^{2}} \\
& =\frac{24 \delta^{4} \theta^{2} \lambda^{2} a^{2}+24 \delta^{3} \theta^{3} \lambda(1-\lambda) a^{2}+6 \delta^{2} \theta^{4}(1-\lambda)^{2} a^{2}}{16 \delta\left(\delta^{2}-\theta^{2}\right)\left(4 \delta^{2}-\theta^{2}\right)^{2}} .
\end{aligned}
$$

Besides, by $\lambda>1 / 2$ we have

$$
\begin{aligned}
\max (U+V) & -\max U-\max V \\
& =\frac{4 \delta^{4} \theta^{2}\left[\lambda^{2}+(1-\lambda)^{2}\right] a^{2}+16 \delta^{3} \theta^{3} \lambda(1-\lambda) a^{2}+5 \delta^{2} \theta^{4}\left[\lambda^{2}+(1-\lambda)^{2}\right] a^{2}+2 \delta \theta^{5} \lambda(1-\lambda) a^{2}}{16 \delta\left(\delta^{2}-\theta^{2}\right)\left(4 \delta^{2}-\theta^{2}\right)^{2}} \\
& <\frac{9 \delta^{4} \theta^{2} \lambda^{2} a^{2}+9 \delta^{4} \theta^{2}(1-\lambda)^{2} a^{2}+18 \delta^{3} \theta^{3} \lambda(1-\lambda) a^{2}}{16 \delta\left(\delta^{2}-\theta^{2}\right)\left(4 \delta^{2}-\theta^{2}\right)^{2}} \\
& <\frac{18 \delta^{4} \theta^{2} \lambda^{2} a^{2}+18 \delta^{3} \theta^{3} \lambda(1-\lambda) a^{2}}{16 \delta\left(\delta^{2}-\theta^{2}\right)\left(4 \delta^{2}-\theta^{2}\right)^{2}} .
\end{aligned}
$$

Hence, the value of $[\max (Z+U)-\max Z-\max U]$ is larger than the value of $[\max (U+V)-\max U-\max V]$ under $\delta=2 \theta$. 
Moreover, the proportion of the market share also plays an important role by comparing the above expressions.

Given the above, whether the value of

$$
[\max (U+V)-\max U-\max V]-[\max (Z+U)-\max Z-\max U]
$$

is positive or negative depends on $\lambda, \delta$ and $\theta$.

\section{Appendix B.}

The proof for the following two conclusions:

(1) When $(Z, U)$ is the optimum two-player coalition, the difference between $V^{*}$ and $\max V$ depends on $\delta, \theta$, and $\lambda$.

(2) When $(U, V)$ is the optimum two-player coalition, $Z^{*}$ is higher than max $Z$, i.e., the final revenue of the manufacturer is still higher than the one he gains in the complete competition situation, though he is not a member of the optimum two-player coalition.

Proof. (1) We consider the conclusion with regard to $V^{*}$ (given by (5.34)) and $\max V$ (given by (4.12)). The difference between $\max (Z+U+V)$ and $\max (Z+U)$ is

$$
\max (Z+U+V)-\max (Z+U)=\frac{(1-\lambda)^{2} a^{2}}{8 \delta} .
$$

By (5.34), the value of $V^{*}$ is then obtained as follows:

$$
V^{*}=\frac{(1-\lambda)^{2} a^{2}}{12 \delta}
$$

By comparing $V^{*}$ and $\max V$, we have

$$
V^{*}-\max V=\frac{\left[4 \delta^{4}(1-\lambda)^{2}-12 \delta^{3} \theta \lambda(1-\lambda)-3 \delta^{2} \theta^{2} \lambda^{2}-8 \delta^{2} \theta^{2}(1-\lambda)^{2}+\theta^{4}(1-\lambda)^{2}\right] a^{2}}{12 \delta\left(4 \delta^{2}-\theta^{2}\right)^{2}} .
$$

By the above expression we can see, whether the difference between $V^{*}$ and $\max V$ is positive or negative, depends on $\delta, \theta$, and $\lambda$.

(2) First, the difference between $\max (Z+U+V)$ and $\max (U+V)$ is given as follows:

$$
\max (Z+U+V)-\max (U+V)=\frac{3 \delta \lambda^{2} a^{2}+3 \delta(1-\lambda)^{2} a^{2}+6 \theta \lambda(1-\lambda) a^{2}}{16\left(\delta^{2}-\theta^{2}\right)} .
$$

By (5.35), the value of $Z^{*}$ is then obtained as follows:

$$
Z^{*}=\frac{7 \delta \lambda^{2} a^{2}+7 \delta(1-\lambda)^{2} a^{2}+14 \theta \lambda(1-\lambda) a^{2}}{48\left(\delta^{2}-\theta^{2}\right)} .
$$

By comparing $Z^{*}$ and $\max Z$, we have

$$
Z^{*}-\max Z=\frac{\left(4 \delta^{3}-19 \delta \theta^{2}\right)\left[\lambda^{2}+(1-\lambda)^{2}\right] a^{2}-\left(16 \delta^{2} \theta+14 \theta^{3}\right)(1-\lambda) \lambda a^{2}}{48\left(4 \delta^{2}-\theta^{2}\right)\left(\delta^{2}-\theta^{2}\right)} .
$$

By the above expression we can see, whether the difference between $Z^{*}$ and max $Z$ is positive or negative, depends on $\delta, \theta$, and $\lambda$.

In reality, by the expressions, $V^{*}$ (or $Z^{*}$ ) may be larger than max $V$ (or max $Z$ ) only when $\delta$ is great larger than $\theta$.

Acknowledgements. We would like to thank the editors and reviewers for their constructive suggestions and corrections to enhance the clarity of the present article. 


\section{REFERENCES}

[1] G.S. Cai, Channel selection and coordination in dual-channel supply chains. J. Retailing 86 (2010) 22-36.

[2] G.S. Cai, Z.G. Zhang and M. Zhang, Game theoretical perspectives on dual-channel supply chain competition with price discounts and pricing schemes. Int. J. Prod. Econ. 117 (2009) 80-96.

[3] E.B. Cao, Y.J. Ma and C. Wan, Contracting with asymmetric cost information in a dual-channel supply chain. Oper. Res. Lett. 41 (2013) 410-414.

[4] J. Chen, H. Zhang and Y. Sun, Implementing coordination contracts in a manufacturer Stackelberg dual-channel supply chain. Omega 40 (2012) 571-583.

[5] W.K. Chiang, D. Chhajed and J.D. Hess, Direct marketing, indirect profits: a strategic analysis of dual-channel supply-chain design. Manage. Sci. 49 (2003) 123-142.

[6] A. Dumrongsiri, M. Fan and A. Jain, A supply chain model with direct and retail channels. Eur. J. Oper. Res. 187 (2008) 691-718.

[7] P. He, Y. He and H. Xu, Buy-online-and-deliver-from-store strategy for a dual-channel supply chain considering retailer's location advantage. Transp. Res. Part E 144 (2020) 102127.

[8] Y. He, H. Huang and D. Li, Inventory and pricing decisions for a dual-channel supply chain with deteriorating products. Oper. Res. 20 (2020) 1461-1503.

[9] S. Huang, C. Yang and X. Zhang, Pricing and production decisions in dual-channel supply chains with demand disruptions. Comput. Ind. Eng. 62 (2012) 70-83.

[10] H. Huang, H. Ke and L. Wang, Equilibrium analysis of pricing competition and cooperation in supply chain with one common manufacturer and duopoly retailers. Int. J. Prod. Econ. 178 (2016) 12-21.

[11] H. Jafari, S.R. Hejazi and M. Rasti-Barzoki, Pricing decisions in dual-channel supply chain with one manufacturer and multiple retailers: a game-theoretic approach. RAIRO:OR 51 (2017) 1269-1287.

[12] B. Li, P.W. Hou and P. Chen, Pricing strategy and coordination in a dual channel supply chain with a risk-averse retailer. Int. J. Prod. Econ. 178 (2016) 154-168.

[13] M.Q. Liu, E.B. Cao and C.K. Salifou, Pricing strategies of a dual-channel supply chain with risk aversion. Transp. Res. Part E 90 (2016) 108-120.

[14] Q.H. Lu and N. Liu, Pricing games of mixed conventional and e-commerce distribution channels. Comput. Ind. Eng. 64 (2013) $122-132$.

[15] L.D. Ma, R. Zhang and S.D. Guo, Pricing decisions and strategies selection of dominant manufacturer in dual-channel supply chain. Econ. Modelling 29 (2012) 2558-2565.

[16] Z. Pi, W. Fang and B. Zhang, Service and pricing strategies with competition and cooperation in a dual-channel supply chain with demand disruption. Comput. Ind. Eng. 138 (2019) 106130.

[17] J.K. Ryan, D. Sun and X.Y. Zhao, Coordinating a supply chain with a manufacturer-owned online channel: a dual channel model under price competition. IEEE Trans. Eng. Manage. 60 (2013) 247-259.

[18] B. Rodriguez and G. Aydin, Pricing and assortment decisions for a manufacturer selling through dual channels. Eur. J. Oper. Res. 242 (2015) 901-909.

[19] S. Saha, S. Majumder and I.E. Nielsen, Is it a strategic move to subsidized consumers instead of the manufacturer? IEEE Access 7 (2019) 169807-169824.

[20] H. Sun, Y. Wan, Y. Li, L. Zhang and Z. Zhou, Competition in a dual-channel supply chain considering duopolistic retailers with different behaviours. J. Ind. Manage. Optim. 17 (2021) 601-631.

[21] C. Tang, H. Yang, E. Cao and K.K. Lai, Channel competition and coordination of a dual-channel supply chain with demand and cost disruptions. Appl. Econ. 50 (2018) 4999-5016.

[22] A. Tsay and N. Agrawal, Channel conflict and coordination in the E-commerce age. Prod. Oper. Manage. 13 (2004) 93-110.

[23] B. Yan, T. Wang and Y. Liu, Decision analysis of retailer-dominated dual-channel supply chain considering cost misreporting. Int. J. Prod. Econ. 178 (2016) 34-41.

[24] D.Q. Yao and J.J. Liu, Competitive pricing of mixed retail and e-tail distribution channels. Omega 33 (2005) $235-247$.

[25] P. Zhang, Y. He and C.M. Shi, Retailer's channel structure choice: online channel, offline channel, or dual channels? Int. J. Prod. Econ. 191 (2017) 37-50.

[26] P. Zhang, Y. He and X. Zhao, "Preorder-online, pickup-in-store" strategy for a dual-channel retailer. Transp. Res. Part E: Logistics Transp. Rev. 122 (2019) 27-47. 